Sorda \& Sonora (1) 2018: 24 - 37

DOI: https://doi.org/10.18800/sordaysonora.201801.002

\title{
Sobre los sujetos vacíos en español
}

\author{
On Spanish null subjects
}

\author{
Francisco Cerna-Herrera \\ Pontificia Universidad Católica del Perú \\ francisco.cerna@pucp.pe
}

\section{RESUMEN}

El presente trabajo es una aplicación del 'Principio de Proyección' y del 'Principio de Proyección Extendida' a una oración existencial en español. Con el fin de, primero, saturar los marcos de subcategorización y, luego, señalar los distintos sujetos de la oración analizada, es que se revisan los tipos de sujetos vacíos (pro-pequeño y PRO-grande) en la oración y algunos aspectos de los verbos y las raíces verbales que la componen, de manera que se cumpla con los requisitos que plantean los dos principios mencionados. Hecho esto, se presentan ciertos fenómenos sobre la construcción de la oración que merecen estudios futuros.

Palabras claves: 'Principio de Proyección', 'Principio de Proyección Extendida', sujetos vacíos en español, oraciones existenciales en español

\section{ABSTRACT}

The present paper is an application of the 'Projection Principle' and the 'Extended Projection Principle' to an existential sentence of Spanish. With the purpose of, first, fulfill the subcategorization frames and, then, show the different 
subjects of the analyzed sentence, the types of null subjects (small-pro and bigPRO) are studied within the sentence and some of the aspects of the verbs and the verbal roots that compose it, so that the requirements posed by the mentioned principles are accomplished. Once this is done, certain phenomenon about the construction of the sentence which must be studied further are presented. Keywords: 'Projection Principle', 'Extended Projection Principle', null subjects in Spanish, existential sentences in Spanish 


\section{o. Antecedentes}

En 1982, Chomsky propuso el así llamado 'Projection Principle' (PP, 'Principio de Proyección'): “One is the projection principle, already mentioned, which states that lexical structure must be represented categorically at every syntactic level” (1982a: 84). Lo copio (1) según lo presenta informalmente Montalbetti (12).

(1) Projection Principle.

Lexical requirements must be met at every level.

Esta es la propuesta de que los requerimientos léxicos de un ítem (léxico) deben representarse en la sintaxis de tal manera que se saturen sus marcos de subcategorización (cf. Chomsky 1965, Montalbetti 1984, Marantz 1981).

Después se complementa al PP con el 'Extended Projection Principle' (EPP, 'Principio de Proyección Extendida'), como sigue:

It seems, then, that the requirement that a clause have a subject position is independent of the Projection Principle. We may think of it instead as a general principle governing D-structures, hence also governing structures derived from them. [...] The Projection Principle and the requirement that clauses have subjects are conceptually quite closely related. In fact, we may stipulate that a lexical element that $\theta$-marks its subject position (e.g. persuade but not seem) does so if and only if the position is filled, that is, in clauses but only optionally in NPs [...] I will henceforth refer to the Projection Principle long with the requirement that clauses have subjects as the Extended Projection Principle. (Chomsky 1982b: 10)

(2) Extended Projection Principle: the requirement that clauses have subjects. Esto ocasiona que incluso ante la ausencia de un sujeto léxico, la posición de sujeto deba ser ocupada ya que se ha planteado el EPP como la obligatoriedad de los sujetos.

La consecuencia del principio de proyección es la necesidad de que "la selección categorial de un predicado se refleje en todos los niveles de representa- 
ción gramatical"; debemos siempre ser capaces de identificar los argumentos de un determinado ítem léxico (Bosque y Gutiérrez-Rexach 344).

\section{Los sujetos vacíos ${ }^{1}$}

Se han propuesto dos elementos, uno de los cuales debe ocupar la posición-sujeto sin realizarse ni léxica ni fonológicamente (y, precisamente, para "solucionar" la aparente falta de sujeto en construcciones donde estas realizaciones no son permitidas): pro y $P R O$ (small-pro y big-PRO).

Por su parte, pro-pequeño o small-pro es aquel sujeto vacío que aparece en el siguiente tipo de oraciones:

(3) pro caminó

pro come-PST.3sg

pro es, entonces, un pronombre no pronunciado, pero definido, que aparece en la posición de sujeto, el cual fue primero propuesto en Chomsky (1982) (Brucart y MacDonald 579).

$P R O$, por otro lado, es otro pronombre no pronunciado de distinta clase:

(4) Juan quería PRO comer.

Juan want-PST-3sg.

PRO eat.

Ambos son pronombres no pronunciados, pero muestran variadas diferencias: mientras que pro aparece en contextos finitos, PRO está limitado a contextos no finitos (Brucart y MacDonald 580).

\subsection{PRO}

Con respecto a PRO, Chomsky (1981) argumenta que sus propiedades y distribución siguen las condiciones de ligamiento bajo la suposición de que PRO es al mismo tiempo [+pronombre] y [+anáfora] (cf. Jaeggli y Safir 1989); esto nos llevaría a concluir que PRO debe ser ligado y al mismo tiempo libre dentro de su dominio de control. Esto se ha tomado como una razón para concluir que,

1 El razonamiento que en esta sección ha sido planteado en Brucart y MacDonald (2012); uso también ejemplos similares a los ya presentados en ese texto. 
debido a que ambas condiciones contradictorias no pueden ser conciliadas, PRO debe ser un pronombre no-controlado (no puede controlarse):

(5) Teorema sobre PRO: PRO no puede ser controlado. (Jaeggli y Safir 14)

La Inflexión no infinitiva no es un controlador, así como no lo es su posición como el sujeto de una cláusula no finita (Brucart y MacDonald 580). (4) es así un caso en el que el controlador (antecedente) de PRO debe ser el sujeto matriz Juan. Esto ilustra un caso de control obligatorio, en contraste con lo que se conoce como control no-obligatorio como en el siguiente ejemplo:
(6) Juan cree que PRO comer demasiado es malo. Juan think-PRES.3sg that PRO eat too much be-PRES.3sg bad

En ese sentido, muchas versiones de la Teoría de Control proponen que cuando PRO falla en ser controlado (es decir, es libre), es asignado el valor por defecto 'arb': un índice reservado para elementos que pueden estar no-ligados y a los que les puede faltar una referencia específica e independiente (Authier 45). Authier (45) propone los siguientes ejemplos en francés:

(7) a. [PROarb chasser à l'arc] est à la mode cette année.

To hunt with a bow is fashionable this year.

PROarb Cazar con un arco está a la moda este año.

b. La peau de ce sanglier est bien trop épaisse pour

The skin of this boar is way too thick

La piel de ese jabali es demasiado gruesa para

[PROarb la découper au canif]

to carve it with a pocker knife.

PROarb tallar(la) con un cuchillo de bolsillo. 
Allí donde PRO no encuentra un potencial controlador, obtiene el índice 'arb'.

\section{2 pro}

\subsection{1 proexpletivo}

Por otra parte, existe un tipo de pro que es necesario explicar para el presente estudio. Es aquel de las siguientes oraciones:

(8) a. pro Parece que Juan llegará tarde.

b. It seems that John will arrive late.

c. pro Lloverá mañana.

d. It will rain tomorrow.

Presento estas oraciones en pares puesto que quiero comparar el contraste entre lenguas morfológicamente ricas (cf. Bosque y Gutiérrez-Rexach 2009) que aceptan la presencia de un pro en el tipo de construcciones que he presentado con aquellas lenguas con una morfología "pobre" (en el sentido en el que requiere explicitar el sujeto léxicamente). El español es una de estas lenguas y el inglés no, por lo que en inglés se resuelve el sujeto nulo con un It como se puede ver en ( $8 \mathrm{~b}$ ) y (8d). Este pro que tiene rasgos de tercera persona y posee el mismo contenido que sus contrastes en lenguas morfológicamente pobres (It en inglés e $I l$ en francés, por ejemplo), es el que se presenta en contextos de verbos meteorológicos como (8c) y verbos impersonales como (8a). Este tipo de pro se conoce como pronombre pleonástico o expletivo; estos no saturan ningún argumento y carecen de contenido semántico. En español, precisamente debido a que carecen de contenido semántico, no pueden funcionar como pronombres expletivos (cf. Bosque y Gutiérrez-Rexach 2009):

(9) a. *Él/*Eso/*Ello parece que Juan llegará tarde.

b. *Él/*Eso/*Ello lloverá mañana.

En español, entonces, existe un pronombre nulo expletivo: pro expletivo, en adelante, pro $_{\text {expletivo }}$. 


\subsection{2 pro $_{\text {existencial }}$}

Por último, existe un tipo más de pro que me interesa: el de tipo existencial. Aquí hay dos posturas. Quienes argumentan en favor de la necesidad de un sujeto para cada oración (en el sentido de especificador en una estructura sintáctica, la posición-sujeto), aceptan y de hecho requieren la existencia de este tipo de pro. Por otro lado, también hay quienes critican la existencia del pro ${ }_{\text {existencial, }}$, como Rodríguez-Mondoñedo (2006).

El trabajo mencionado marca una diferencia dialectal en español dentro de lo que se denominan 'Spanish Existential Constructions' (SEC, 'Construcciones Existenciales del Español): en un primer dialecto, 'Spanish Dialect I’ (SI), el verbo (del controvertido pro existencial) tiene una conjugación predeterminada (por 'default'), que es tercera persona-singular; mientras que en el segundo dialecto, SII, el mismo verbo se conjuga con respecto al acusativo nominal, inclusive si este es un clítico (326-327).

(10) a. SI: verbo (existencial) $\rightarrow$ tercera persona-singular

Hay puertas cerradas.

IS DOORS CLOSED.

There are closed doors.

b. SII: verbo (existencial) $\rightarrow$ asimila la conjugación del ACC nominal.

Las hay.

CL-ACC is.

En Rodríguez-Mondoñedo (2006) se argumenta que, en las construcciones existenciales del español, aquellas que usan al verbo Haber, el nominal no ocupa la posición-sujeto: en términos sintácticos, no hay algo que sature esta posición. De todas maneras, aquí marcaré la posición-sujeto con un pro en las construcciones existenciales, solo para contrastar esta instancia (este pro) con los otros de la oración a analizar: solo es una cuestión metodológica. La existencia o no de este tipo de pro está fuera de los alcances de este trabajo, aún si se ha argumentado en contra de este elemento en el artículo mencionado anteriormente. 


\section{Las puertas prohibidas}

La teoría de los sujetos vacíos o nulos presenta una serie de controversias y preguntas, además de las ya exploradas. Quiero discutir algunas cuestiones acerca de esta teoría a propósito de las siguientes oraciones:

(11) a. Hay puertas que está prohibido abrir.

b. Hay puertas que está prohibido volver a abrir.

Hay distintos aspectos de estas oraciones que las vuelven interesantes:

1. En ambas el sujeto no se presenta léxicamente ni puede hacerlo.

2. En (11b) hay cuatro verbos (más precisamente, dos verbos: 'hay' y 'está'; dos raíces verbales: 'volver' y 'abrir'; y un participio: 'prohibido').

3. Los dos verbos antes mencionados están conjugados por igual: tercera persona, singular.

En este trabajo adopto la necesidad de saturar la posición-sujeto (el especificador del ST, el sintagma de tiempo), lo que me permite analizar estas oraciones en cuanto a sus posiciones-sujeto no realizadas léxica ni fonológicamente:

(12) a. $[p r o]_{\mathrm{i}}$ Hay $[\text { puertas }]_{\mathrm{k}}$ que $[\text { pro }]_{*_{i j}}$ está prohibido $[P R O]_{\mathrm{j}}[\mathrm{abrir}]_{\mathrm{k}}$.

b. $[p r o]_{\mathrm{i}}$ Hay $[\text { puertas }]_{\mathrm{k}}$ que $[p r o]_{*_{\mathrm{i} j}}$ está prohibido $[P R O]_{\mathrm{j}^{* * 1}}$ volver a $[P R O]_{\mathrm{j}}$ $[\mathrm{abrir}]_{\mathrm{k}}$.

En ambas oraciones hay una indexación prohibida entre el primer pro, el de 'hay', y el segundo pro, el de 'está'. Según he presentado en la sección anterior, el primero es un proexistencial que surge del verbo existencial 'haber' y el segundo es un proarbitrario debido a que no encuentra un controlador.

Por otra parte, en (12b), hay una cierta estructura argumental de la raíz verbal 'volver' que debe ser analizada por sus efectos con respecto a los sujetos vacíos, por lo que la presento en (13):

(13) $[\text { volver }]_{2}=\{$ Agente, Estado $\}$

En principio, (13) es lo primero que se me ocurre para la raíz verbal 'volver'. Hay un hecho que no puedo esclarecer y ese es la 'a' que acompaña a la raíz verbal aquí analizada, pero que, ciertamente, modifica la estructura del argumento. 
Hay, sin embargo, un apunte importante que hacer aquí. Este 'volver a', al menos en la oración analizada en (11b), no tiene un sentido de "regresar" como en (14a), sino un sentido-de-repetición (o como quiera llamarse) como en (14b).

(14) a. Juan volvió a casa.

= Juan no estaba en casa y regresó a casa.

b. Juan volvió a beber.

= Juan no bebía, pero lo hizo de nuevo (una o más de una vez).

Este 'volver a' como repetición es el que se encuentra en la oración (12b). En las dos últimas oraciones que he presentado hay un 'volver a', pero la diferencia radica en la función que realiza este elemento: mientras que en (14a) el argumento externo se interpreta como 'dirección' (el argumento externo es el lugar a donde se regresa), en (14b) la interpretación es 'actividad que se vuelve a hacer'.

Entonces, una correcta estructura argumental de la raíz verbal 'volver a' sería la siguiente:

(15) En la oración:

$[\text { pro }]_{\mathrm{i}}$ Hay puertas que $\left[\mathrm{pro}_{*_{\mathrm{*} / \mathrm{j}}}\right.$ está prohibido $[\mathrm{PRO}]_{\mathrm{j} /{ }^{* * \mathrm{k}}}$ volver a $[\mathrm{PRO}]_{\mathrm{j}}$ abrir.

$$
\begin{aligned}
& {[\text { volver a }]_{2}=\left\{P R O_{\mathrm{j}},[\text { abrir }]_{2}\right\}} \\
& {[\text { abrir }]_{2}=\left\{P R O_{\mathrm{j}} \text {, las puertas }\right\}}
\end{aligned}
$$

He añadido también la estructura de 'abrir', que, como se ve, ocupa uno de los roles temáticos de 'volver a', sin que eso signifique que sus propios roles argumentales no deban ser también saturados. Todo esto explica la necesaria indexación del $P R O$ de 'volver' con el $P R O$ de 'abrir', que, si bien obvia, no es tan sencilla de explicar. Quien ocupe la posición de sujeto o el rol de agente de 'volver a' debe también ocupar la misma posición en la raíz verbal 'abrir'.

Lo que sucede con este 'volver' en (15) ha sido plantado en Laca (2004). Laca (425) propone que en las lenguas romances hay una variedad de construcciones que contienen un infinitivo o un gerundio que actúa como un "predicador principal” y, al mismo tiempo, un verbo mayor. En el caso de nuestro ejemplo, el predicador principal sería 'abrir' y el otro verbo 'volver a'. La auto- 
ra establece que el verbo mayor es o bien un "aspectualizador" [i.e. un verbo superléxico que no denota una eventualidad sino una parte de la estructura temporal de cualquier eventualidad, como 'empezar (a)' o 'terminar (de)] o un verbo de movimiento o locación (como 'ir', 'seguir', 'volver'); estas construcciones son denominadas "perífrasis aspectuales" (o "perífrasis de aspecto”). Estas "perífrasis aspectuales" son, hasta cierto punto, recursivas, de manera que más de un verbo mayor puede ser asociado con un solo predicador:

(16) a. Juan acaba de dejar de fumar.

b. María ha vuelto a empezar a cantar.

El verbo finito/mayor parece ser semánticamente "defectuoso", por lo que la construcción entera "hereda" la estructura argumental y las restricciones de selección del predicador principal (Laca 426); como fue adelantado en (15).

En la tabla que presento a continuación, se encuentra el problemático 'volver a', que es una perífrasis modificadora de evento (o eventualidad): este elemento, pues, modifica el evento de 'abrir', el cual, además, hereda algunos de sus rasgos [su sujeto, como hemos explicado en (15) y según el razonamiento de Laca (2004)]. 


\begin{tabular}{|c|c|c|c|c|c|c|c|c|c|c|}
\hline \multirow{6}{*}{ 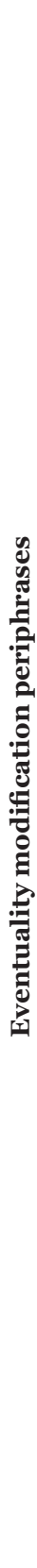 } & 胥 & 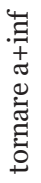 & 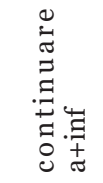 & 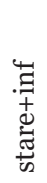 & 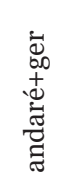 & 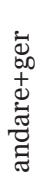 & 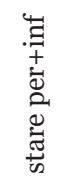 & 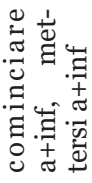 & 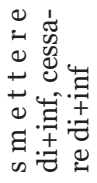 & 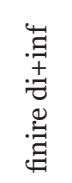 \\
\hline & 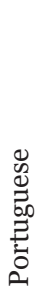 & 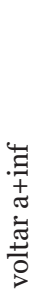 & 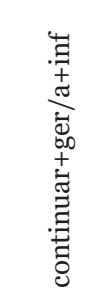 & & 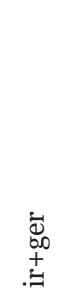 & 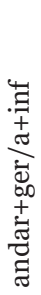 & 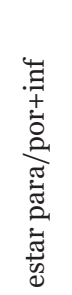 & 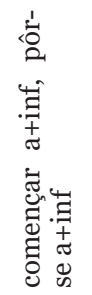 & 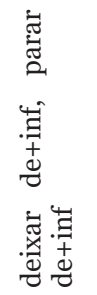 & 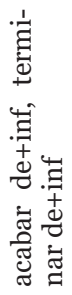 \\
\hline & 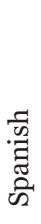 & $\begin{array}{l}\Psi \\
. \Xi \\
+ \\
\pi \\
\dot{0} \\
D \\
0 \\
0\end{array}$ & 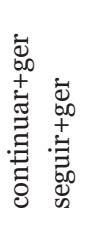 & & 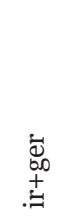 & 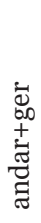 & 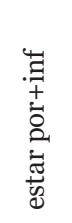 & 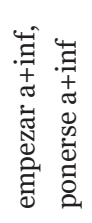 & 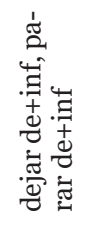 & 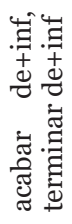 \\
\hline & 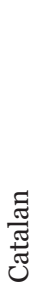 & 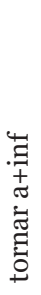 & 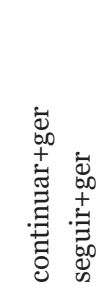 & & 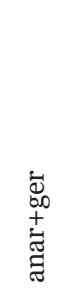 & 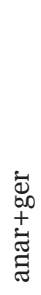 & 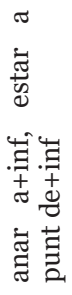 & 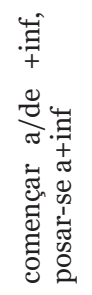 & 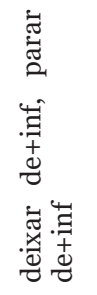 & 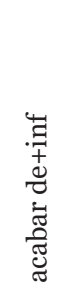 \\
\hline & 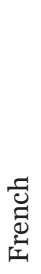 & & 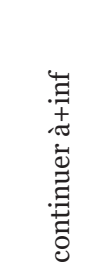 & & & & 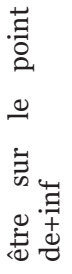 & 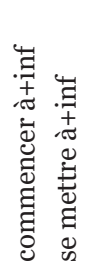 & 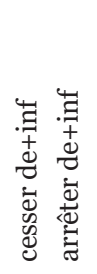 & 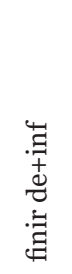 \\
\hline & & 离 & 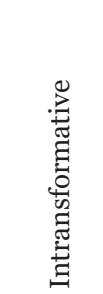 & 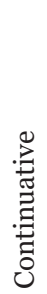 & 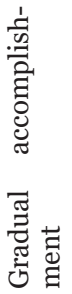 & 葛 & 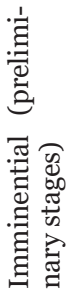 & 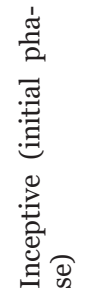 & 离 & $\frac{\stackrel{D}{0}}{\frac{0}{0}}$ \\
\hline
\end{tabular}


Según la tabla anterior, entonces, la modificación eventual que 'volver a' ocasiona en el evento 'abrir' corresponde a repetición / restitución.

Esta sería la razón por la que 'volver a' y 'abrir' comparten el mismo sujeto PRO, como suponía en (15), que termina por ser una postulación correcta.

Otro hecho que resulta de interés aquí es el análisis de pro con respecto a la conjugación del verbo y su participio:

(17) a. *Hay puertas que están prohibido (volver a) abrir.

b. *Hay puertas que están prohibidas (volver a) abrir.

En (17a), por ejemplo, se concuerda puertas con el verbo estar. Esto da como resultado una oración a todas luces agramatical. Peor aún si, como en (17b), también se concuerda 'prohibidas'.

Esta prueba nos ayuda a clarificar (si es que de principio no es claro) que ni el verbo ni el participio se conjugan con respecto al elemento puertas, que, entonces, no es el sujeto. La conjugación es de tercera persona, singular; esto es

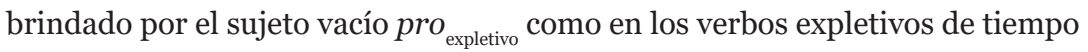
(llover, por ejemplo).

(18) a. Hay niños que está prohibido abandonar.

b. *Hay niños que están prohibidos abandonar.

En los ejemplos de (18) solo se han cambiado los elementos para probar que se trata de un mecanismo de la construcción, no dependiente de sus elementos.

Hay una forma de obtener gramaticalidad en la oración, lo cual se consigue conjugando la construcción 'estar prohibid-' según el elemento 'puertas' y agregando un 'de' entre esta construcción y abrir, como sigue.

(19) a. ? Hay puertas que están prohibidas de abrir.

b.? Hay niños que están prohibidos de abandonar.

(20) a. ? Hay puertas que están prohibidas de volver a abrir.

Las oraciones en (19) no parecen completamente agramaticales, pero, al menos a mí, se me hacen extrañas (anómalas).

Un hecho notorio aquí es la necesidad del 'de'. Como he marcado en las oraciones de (19) y en la de (20), la conjugación de la construcción 'est(a)prohibid-' según 'puertas' exige la aparición de este elemento, sin el cual las oraciones son agramaticales. 
Finalmente, eliminar el primer verbo de las oraciones no arregla su situación, pero disminuye el fenómeno a la construcción 'est(a)- prohibid-'.

(21) a. Está prohibido abrir estas/las/esas/ø puertas.

b. Está prohibido abandonar estos/los/esos/ø niños.

\section{Conclusiones}

1. Laca (2004) brinda una explicación al fenómeno de herencia de papeles temáticos entre 'volver a' y 'abrir'. En (15) hemos visto que ambos eventos necesariamente tienen el mismo sujeto, el mismo PRO en el caso de nuestra oración. Esto también se debiera analizar en términos de las construcciones denominadas 'antipasivas', donde también hay modificaciones con respecto al agente de las estructuras de evento (cf. Rodríguez Ramalle 2005 y 2015).

2. Por un lado, es bastante obvio que los intentos por conjugar la construcción 'est(a)- prohibid-' según el elemento 'puertas' son nulos si es que se deja de lado la necesidad de conjugar toda la construcción. Los rasgos gramaticales de 'prohibid-' son los mismos que los de 'est(a)-' y la razón es bastante sencilla: el primer elemento es participio del segundo. No es tan claro, por otro lado, las consecuencias que esta conjugación tiene en la construcción de la oración Hay puertas que están prohibidas (*de) volver a abrir. El 'de' que aparece se vuelve un elemento obligatorio al momento de la conjugación mencionada, sin que haya una explicación transparente al respecto.

3. Tampoco hay una explicación para la 'a' presente en 'volver a'. En principio, supondré que se trata de un fósil del 'volver a' con sentido de dirección ('volver a casa') que se ha traspasado al 'volver a' de la oración analizada aquí, que tiene sentido de repetición.

4. No ha sido explorado en este trabajo, pero también se deben estudiar, a futuro, los efectos de la subordinación del 'que' en la oración estudiada. Para ello, se requeriría de un árbol sintáctico de X-barra de esta oración que diera cuenta de las múltiples indexaciones internas en la oración, las que he atendido en (15). 


\section{Referencias bibliográficas}

Authier, J-Marc P. "Arbitrary null objects and unselective binding." The null subject parameter. Osvaldo Jaeggli y Kenneth J. Safir (edit.). Kluwer Academic Publishers, 1989. $45-67$.

Bosque, Ignacio, and Javier Gutiérrez-Rexach. Fundamentos de sintaxis formal. Ediciones Akal, 2009.

Brucart, Josep y Jonatahn MacDonald. "Empty Categories and Ellipsis”. The Handbook of Hispanic Linguistics. José Ignacio Hualde, Antxon Olarrea, y Erin O'Rourke (edit.). Blackwell Publishing Ltd 2012. 579-601. https://doi.org/10.1002/9781118228098.ch27 Chomsky, Noam. Lectures on government and binding: The Pisa lectures. Walter de Gruyter, 1993. https://doi.org/10.1515/9783110884166

---. Knowledge of Language: Its Nature, Origin and Use. Praeger, 1982a.

---. Some Concepts and Consequences of the Theory of Government and Binding. MIT Press, $1982 b$.

Jaeggli, Osvaldo, y Kenneth J. Safir. "The null subject parameter and parametric theory". The null subject parameter. Kluwer Academic Publishers, 1989. 1-44. https://doi. org/10.1007/978-94-009-2540-3

Laca, Brenda. "Romance" aspectual” periphrases: eventuality modification versus" syntactic” aspect.” The Syntax of Time. Jacqueline Guéron y Jacqueline Lecarme (edit). The MIT Press 2004. 425-440.

Marantz, Alec. On the nature of grammatical relations. Tesis MIT, 1981.

Montalbetti, Mario. After binding. On the Interpretation of Pronouns. Tesis MIT, 1984.

Rodríguez Ramalle, Teresa. Manual de sintaxis del español. Vol. 4. Editorial Castalia, 2005 .

---. Las relaciones sintácticas. Síntesis, 2015.

Rodríguez-Mondoñedo, Miguel. "Spanish existentials and other accusatives constructions”. Minimalist Essays. Cedric Boeckx (edit.). John Benjamins 2006. 